\section{A Comment on AFM vs. Replicas for High Resolution Imaging}

\author{
Don Chernoff \\ Advanced Surface Microscopy, Inc. \\ donc@asmicro.com
}

High-resolution $\mathrm{Pt} / \mathrm{C}$ shadowing and replication provided important insights into the size and shape of polymers beginning over 40 years ago. The first images of DNA molecules were made this way. However, in my opinion, this methodology has largely been supplanted by the use of Atomic Force Microscopy (AFM), both for direct height measurements (available in $\mathrm{Pt} / \mathrm{C}$ replicas by measuring shadow lengths when the coating is deposited from one direction only) and for imaging molecular contours.

As an everyday example, consider that making a magnetic read and write head for a hard disk drive requires controlling the relative heights of several different regions to a tolerance of about $1 \mathrm{~nm}$. AFM supports production by providing a rapid means of offline analysis, far faster and more precise than any replica method could be.

In the biopolymer area, for more than 10 years it has been relatively easy to prepare dispersions of molecules on smooth surfaces like mica for AFM images. Collagen and DNA are good examples of this.

One way to characterize materials such as collagen is by using Atomic Force Microscopy. Individual molecules can be seen and measured, and whether the molecule is a fragment, monomer, dimer, or higher oligomer can be determined from the length and geometry of the molecule. Monomers are nominally $280 \mathrm{~nm}$ long. Fragments will be shorter. Dimers and higher oligomers may be linear, branched or looped.

Collagen for medical use is commonly produced by digestion of bovine skin. This soluble collagen consists of individual "monomers," longer chains (dimers and higher oligomers) and fragments. In the USA, federal regulations require that the consistency of this mix be documented and controlled. One medical use of purified collagen monomers is to plump up the skin (remove wrinkles). By using AFM to take images of batches of collagen molecules and measuring the molecules within them, one can provide an independent check on other quality assurance techniques, ensure compliance with Federal regulations, and produce better quality materials, figure 1 .
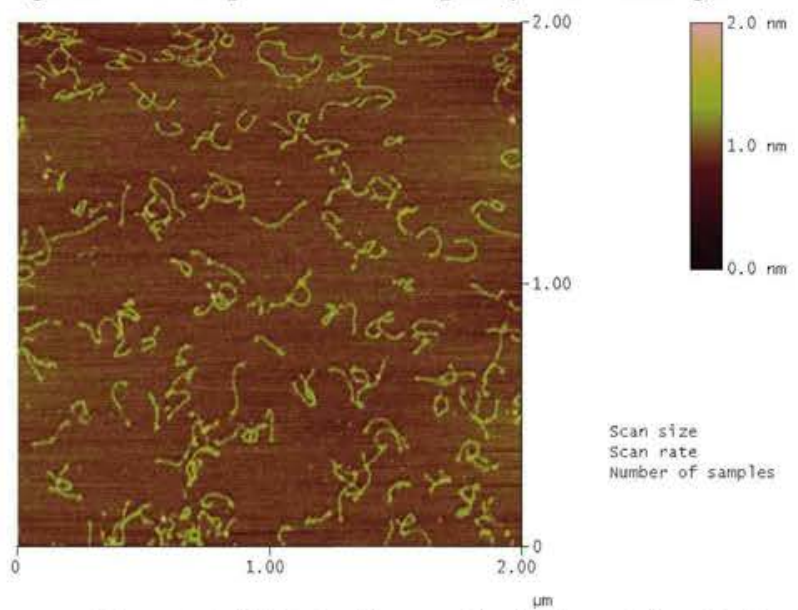

Figure 1: Individual collagen molecules deposited on freshly cleaved mica from an aqueous suspension form wormlike patterns. $2 \mu \mathrm{m}$ scan.
Analysis of DNA is important in medicine and biology. New drugs, new treatments and new understanding of life come from better knowledge of DNA function. This in turn depends on the characteristics of the higher-order structures formed by DNA double helices. Whereas chemical and enzymatic probes yield only the average behavior of an ensemble of DNA molecules, direct observation of individual molecules provides specificity that sharpens insight.

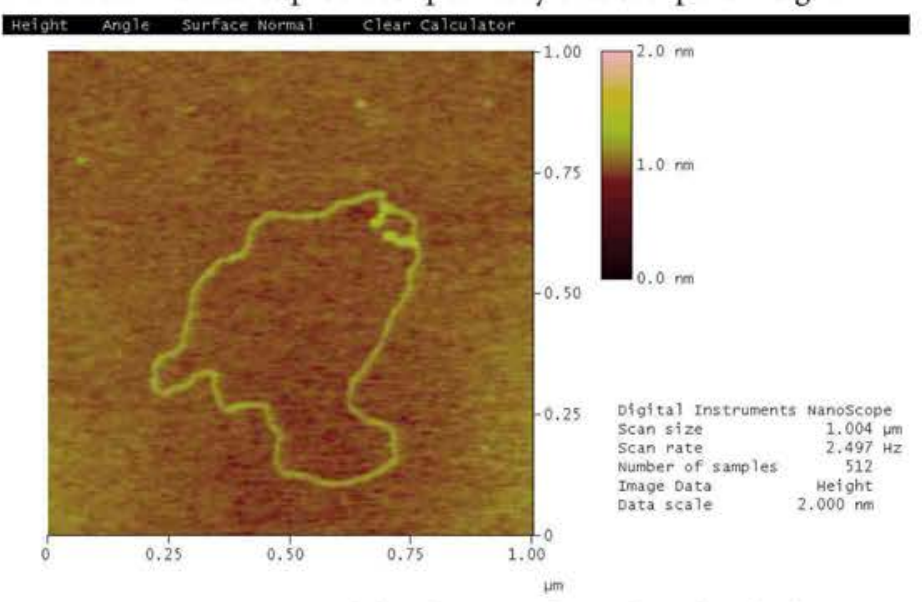

Figure 2: Tapping Mode height image of a single molecule of DNA, dried on a mica surface. Note the bright branch-like segments near the upper right. These thicker, coiled regions are present because the molecule is supercoiled. $1 \mu \mathrm{m}$ scan.

DNA usually has a rod-like conformation that makes it ideal for AFM studies of molecular weight and conformation. Molecular weight is proportional to the contour length of the molecule. The presence of supercoiling in the circular DNA plasmid molecule shown in Figure 2 is evident from the presence of short branch-like features near the upper right corner. The branches are brighter in the height image than other parts of the molecule, which means they are thicker. Such regions are interpreted as coiling of the double helix. The coiling relieves stress and is analogous to the spontaneous coiling of a telephone handset cord.

Sample preparation is fairly straightforward, consisting of preparing dilute solutions of DNA in buffer, applying a drop to a mica surface, rinsing and drying. We usually image using Tapping Mode in air, but liquid tapping is also possible.

Samples for such AFM images are more easily and quickly produced, and provide more accurate dimensional information than can be achieved with replicas.

Disclaimer: ASM provides analytical services using AFM and benefits from increasing the demand for AFM data.

\section{Embedding Cultured Cells Grown in Well Plates}

\author{
Leona Cohen-Gould \\ Cornell University, Ithaca, NY \\ lcgould@med.cornell.edu
}

For years, I have used a hybrid Epon-analog resin to embed in culture dishes. I use a standard Epon formula but utilize the following components: LX-112 and DMP-30 from Ladd Research Industries DDSA and NMA from Electron Microscopy Sciences I know it seems weird, but years ago I tried all sorts things, from the "straight" formulations from each vendor to a bunch of mixtures. This one has 


\section{Precision Lapping Film}

\section{Diamond : Aluminum Oxide - Silicon Carbide}

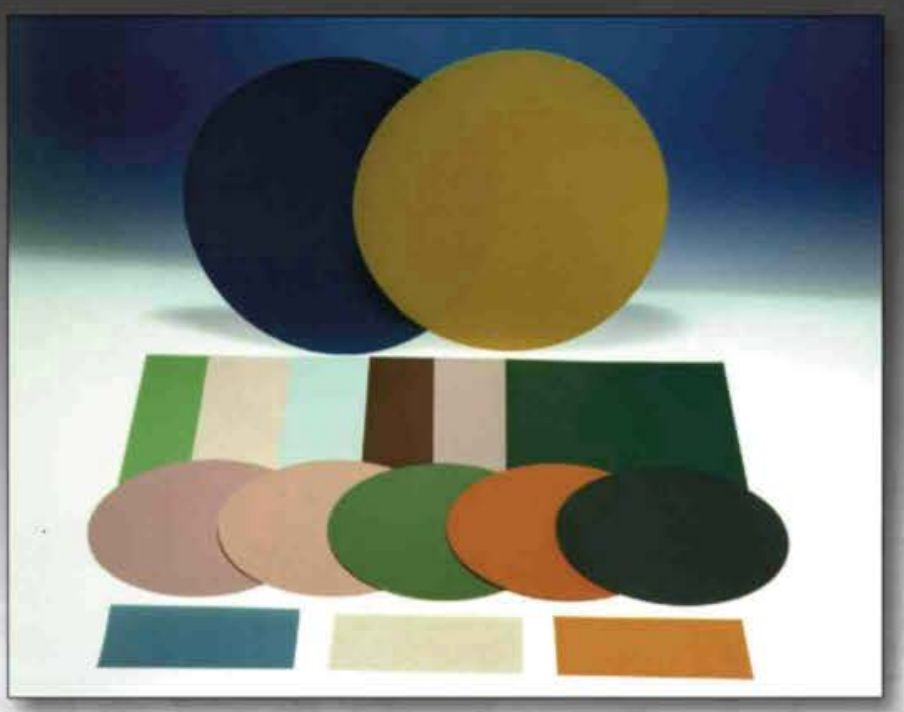

Lapping film consists of precision graded abrasive particles bonded to a flat, uniform polyester film backing. It provides excellent edge retention and maintains planarity across dissimilar materials.

Available Abrasive Types:

Diamond (0.1 to 100 micron)

Silicon Carbide (1.0 to 150 micron)

Aluminum Oxide (0.05 to 150 micron)

\section{Typical Applications}

SEM/TEM preparation with the Tripod Polisher cross-sectioning toot

IC cross-sectioning

Pre-FIB thinning

- Unencapsulated sample polishing

Fiber optic polishing

Metallography

Otolith polishing

\section{Final
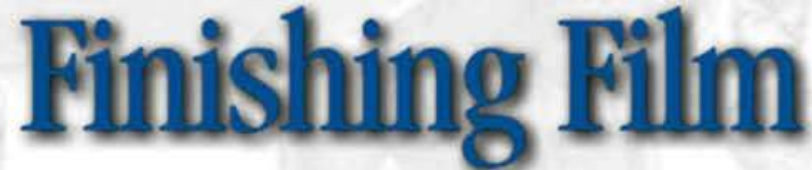

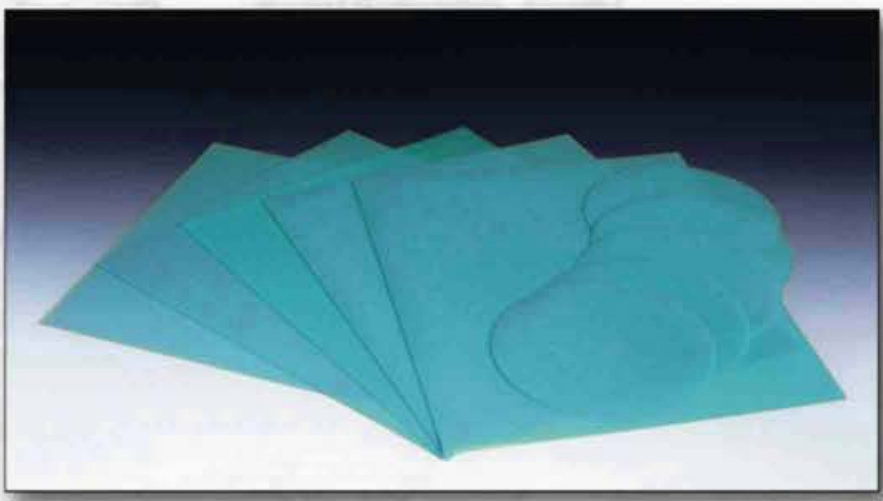

\section{True Blue ${ }^{\mathrm{TM}}$ Film}

True Blue ${ }^{\mathrm{Tu}}$ film is produced with a proprietary process combining a unique mixture of abrasive particle sizes and shapes that are bonded to a film backing which enable the film to produce finer surfaces than are normally found in similarly graded micron films. True Blue ${ }^{\mathrm{TM}}$ film is typically used after the 1 micron diamond lapping film step and provides a flat, nearly scratch free surface.

Standard sizes for all films are $8^{\prime \prime}$ and $12^{\prime \prime}$ diameter, but any size or shape is available. Films are also available with either a plain back or pressure sensitive adhesive (PSA).

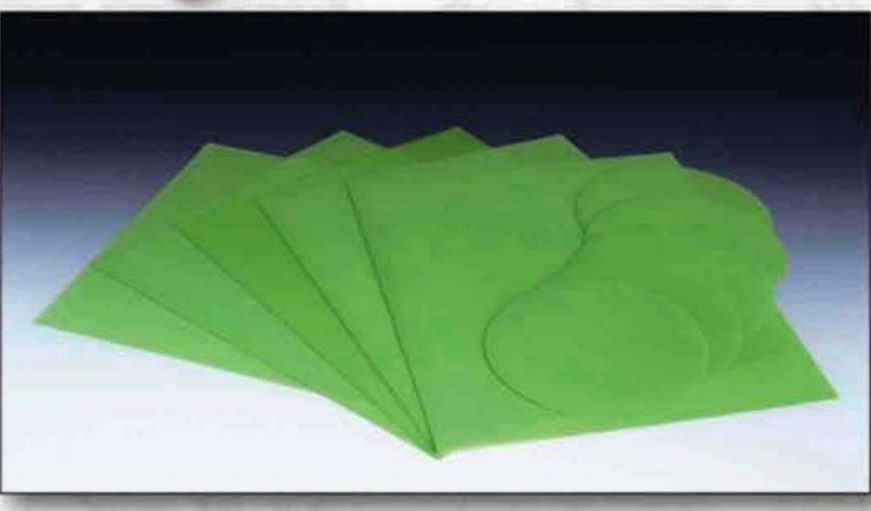

\section{Final Green ${ }^{\mathrm{TM}}$ Film}

Final Green ${ }^{\mathrm{m}}$ film is produced using a unique process which is different from any other type of abrasive film. This process produces a film that is capable of removing even the finest scratches at the final stages of polishing. Final Green ${ }^{\mathrm{m}}$ film is typically used after True Blue $\mathrm{w}^{\mathrm{m}}$ film and produces an ideal sample surface for analysis.

\section{www.southbaytech.com}

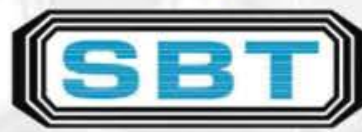

\section{SOUTH BAY TECHNOLOEY, INC.}


never reacted with the plastic of the dishes. Here is how I do the actual embedding of the cell monolayers in the dishes:

1) After the last $100 \%$ ethanol, remove the alcohol and cover the bottom of the well with a layer of resin mixture that is about 2 mm deep.

2) Insert embedding tubes that are made by cutting the pyramidal bottoms off of BEEM capsules (just slice them with a fresh razor blade and be sure to insert them so that the manufactured end rather than the cut one is sitting against the dish).

3) After inserting labels into the tubes, put them into the oven at $60^{\circ}$ overnight.

4) In the morning, fill just the embedding tubes and return everything to the oven again to finish polymerizing.

5) When the resin is cured, grab the tubes with a pair of needlenosed pliers and snap them out. Sometimes a bit of the bottom of the dish comes away with the block, but often a very smooth block face results. If some of the dish comes up, it is easy to see under a dissecting microscope, and the dish portion comes away easily when trimming the block face.

I often cut away part of the block face with a jeweler's saw, either to keep it in reserve or to re-embed it in order to get cross sections, and then trim the rest into a narrow rectangle. When sectioning the resulting block en face, start at $0.25 \mu \mathrm{m}$ (no thick sections), pick up and stain the sections as they are cut. Smooth thin sections should be obtained within a micron. I usually trim a very long rectangle and then start to section in such a way that I am a degree or two off of being perfectly en face from top to bottom, so that I first get sections from one edge of the rectangle and then have a lot of "acreage" to work through if I need more sections later on.

Disclaimer: I have no financial interest in either Ladd or EMS -- I'm just a happy customer who believes in using what works.

\section{Flies in a Box}

\section{Patricia S. Connelly and Linda Ruggiero* \\ *Neuroscience Graduate Program, Oregon Health and Science University, Portland, $O R$ psconnelly@gmail.com,ruggiero@ohsu.edu}

A challenge was presented to us to find a way to support Drosophila melanogaster so that both the anterior and posterior scutellars could be easily visualized under a dissecting microscope and photographed for purpose of measurement. The resulting solution was to utilize a standard grid box with the lid removed. Several holes were filled with Gary's Magic Mountant [1] to just above the rim of the hole. Freshly dead fruitflies were gently lifted with forceps. Then each was placed into a filled hole and both the head and abdomen were pushed into the mountant. The wings were arranged on the plastic on either

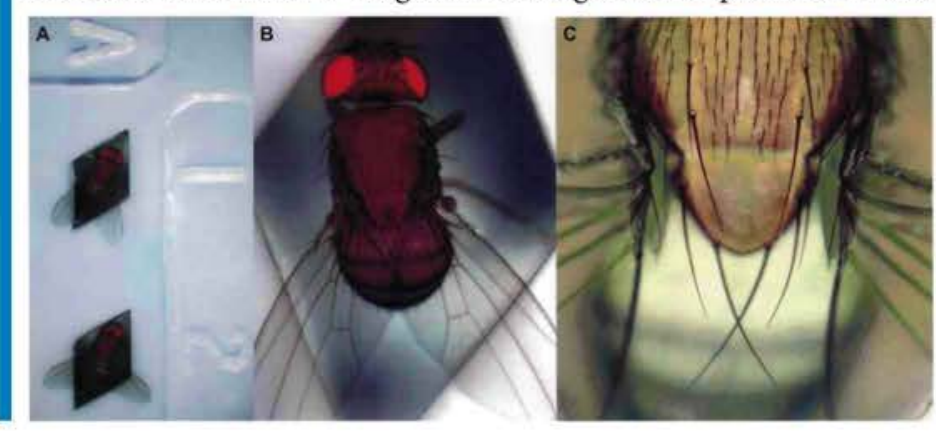

side of the hole as seen in the micrographs.

The best results were obtained if the body of the fly was evenly covered with Gary's Magic Mountant and if the photography was accomplished within a few hours of the death of the fly.

As experiments were being done, it was realized that this technique is also useful to visualize wing form, wing hair information and to study the length and formation of the head and thorax bristles.

\section{Acknowledgements}

The authors wish to thank Dr. Gregory M. Guild, Department of Biology, University of Pennsylvania in whose laboratory the experiments are continuing and Megan Malloy for providing the micrographs.

Gary's Magic Mountant [2]

Dissolve $1.75 \mathrm{~g}$ Canada balsam in $1.0 \mathrm{ml}$ Methylsalicylate (oil of wintergreen)

Stir without heat for about 2 days in a metal container

Pour into glass jar (scintillation vial) and store at $4^{\circ} \mathrm{C}$

[1] Lawrence, P.A., P. Johnson, G. Morata. Methods of marking cells, In Drosophila: A practical approach (ed. D.B. Roberts), pp. 229242. IRL Press, Oxford, 1986.

[2] Ashburner, M. Drosophila A Laboratory Manual, Cold Spring Harbor Laboratory Press, 1989.

\section{A Comment on using FLIM with FRET}

\author{
Karl Garsha \\ Roper Scientific, Tucson, AZ \\ kgarsha@roperscientific.com
}

Depending on the nature of the study and what sort of information one is trying to gather through the use of FRET, FLIM has some compelling advantages in certain situations, and can provide a quantitative evaluation of the donor, acceptor and FRET pair stoichiometry. It does require access to specialized equipment and software. Different approaches to FLIM data acquisition have different strengths and weaknesses. For dynamic studies requiring high time resolution, FLIM acquisition times can fall well short of ideal.

If a yes/no answer to whether FRET is occurring is all that is required, then the polarization anisotropy of the acceptor can be used to determine FRET between fluorescent proteins (Rizzo and Piston, 2005). This is a relatively simple and robust method for confirming the presence/absence of FRET.

A more complex method, also based on polarization anisotropy, is provided by Mattheyses et al. (2004). This polarized FRET technique has the advantage that the pixel-by-pixel concentrations of donor, acceptor and FRET pairs in a manner analogous to FLIM. The advantages to this approach are that it is ostensibly less expensive to implement, and acquisition requires only a single exposure per time point.

When either of the above two approaches to FRET are implemented using widefield illumination in conjunction with a beamsplitting device to permit simultaneous capture of multiple channels using a CCD camera, very high quantum efficiency for detection is possible. All of the pixels are captured in parallel, so noise due to molecules drifting from pixel to pixel during the course of acquisition is minimized if exposures are kept short.

Spectral detection provides advantages as well. Because spectral unmixing can provide correction of the donor bleedthrough into 